\title{
BMJ Open SEPSIS project: a protocol for studying biomarkers of neonatal sepsis and immune responses of infants in a malaria-endemic region
}

\author{
Nadine Fievet, ${ }^{1,2}$ Sem Ezinmegnon, ${ }^{3,4}$ Gino Agbota, ${ }^{5,6}$ Darius Sossou, ${ }^{6}$ \\ Rodolphe Ladekpo, ${ }^{6}$ Komi Gbedande, ${ }^{7}$ Valerie Briand, ${ }^{1}$ Gilles Cottrell, ${ }^{8,9}$ \\ Laurence Vachot, ${ }^{4}$ Javier Yugueros Marcos, ${ }^{4}$ Alexandre Pachot, ${ }^{10}$ \\ Julien Textoris (D) , ${ }^{10,11}$ Sophie Blein, ${ }^{4,10}$ Ulrik Lausten-Thomsen, ${ }^{12}$ \\ Achille Massougbodji, ${ }^{13}$ Lehila Bagnan, ${ }^{6,14}$ Nicole Tchiakpe, ${ }^{6,15}$ \\ Marceline d'Almeida, ${ }^{14,16}$ Jules Alao, ${ }^{17}$ Ida Dossou-Dagba, ${ }^{18}$ Pierre Tissieres (D) , ${ }^{3,12}$ \\ SEPSIS study group collaborators, On behalf of the SEPSIS study group
}

To cite: Fievet N, Ezinmegnon S, Agbota G, et al. SEPSIS project: a protocol for studying biomarkers of neonatal sepsis and immune responses of infants in a malariaendemic region. BMJ Open 2020;10:e036905. doi:10.1136/ bmjopen-2020-036905

- Prepublication history for this paper is available online. To view these files, please visit the journal online (http://dx.doi org/10.1136/bmjopen-2020036905).

Received 10 January 2020 Revised 13 April 2020 Accepted 12 June 2020
Check for updates

(c) Author(s) (or their employer(s)) 2020. Re-use permitted under CC BY-NC. No commercial re-use. See rights and permissions. Published by BMJ.

For numbered affiliations see end of article.

Correspondence to

Professor Pierre Tissieres; pierre.tissieres@aphp.fr

\section{ABSTRACT}

Introduction Neonatal sepsis outreaches all causes of neonatal mortality worldwide and remains a major societal burden in low and middle income countries. In addition to limited resources, endemic morbidities, such as malaria and prematurity, predispose neonates and infants to invasive infection by altering neonatal immune response to pathogens. Nevertheless, thoughtful epidemiological, diagnostic and immunological evaluation of neonatal sepsis and the impact of gestational malaria have never been performed.

Methods and analysis A prospective longitudinal multicentre follow-up of 580 infants from birth to 3 months of age in urban and suburban Benin will be performed. At delivery, and every other week, all children will be examined and clinically evaluated for occurrence of sepsis. At delivery, cord blood systematic analysis of selected plasma and transcriptomic biomarkers (procalcitonin, interleukin (IL)-6, IL-10, IP10, CD74 and CX3CR1) associated with sepsis pathophysiology will be evaluated in all live births as well as during the follow-up, and when sepsis will be suspected. In addition, whole blood response to selected innate stimuli and extensive peripheral blood mononuclear cells phenotypic characterisation will be performed. Reference intervals specific to sub-Saharan neonates will be determined from this cohort and biomarkers performances for neonatal sepsis diagnosis and prognosis tested.

Ethics and dissemination Ethical approval has been obtained from the Comité d'Ethique de la Recherche Institut des Sciences Biomédicales Appliquées (CER-ISBA 85 - 5 April 2016, extended on 3 February 2017). Results will be disseminated through international presentations at scientific meetings and publications in peer-reviewed journals.

Trial registration number ClinicalTrials.gov registration number: NCT03780712.
Strengths and limitations of this study

- This prospective, multicentre study in a limitedresources environment aims to characterise value of neonatal sepsis diagnosis and prognosis biomarkers in a high-risk gestational malaria (GM) area.

- The identified biomarkers through this study could provide new diagnostic and prognostic tools to identify immunosuppressed newborns at risk of neonatal sepsis.

- Clinical and immunological phenotypes, and functional characterisations of a population with high risk of neonatal sepsis brings new insight to understanding the clinical and immune consequences of GM.

- Lack of biological antenatal malaria documentation in the hospital arm may limit the extrapolation to the first and second trimester GM impact.

\section{INTRODUCTION}

Sepsis remains the first cause of perinatal morbidity worldwide affecting more than 3 million neonates every year. ${ }^{1}$ Nearly half of neonatal deaths in sub-Saharan Africa are due to sepsis. ${ }^{2}$ In addition to neonatal sepsis, sub-Saharan Africa and South Asia that encompass $52 \%$ of world global live births, have an overweighed proportion of prematurity, which represents $60 \%$ of all preterm births worldwide. ${ }^{34}$ Low- and middle-income countries (LMIC) are supporting most of these neonatal morbidities and the societal and economic impacts are high, hampering most initiatives that target promotion of neonatal and paediatric health. ${ }^{56}$ Among others factors associated with neonatal infection, frequent endemic parasitic infections 
such as malaria, toxoplasmosis and schistosomiasis affect fetal and perinatal growth and development. Its specific impact on early childhood immunity is recognised and may explain altered vaccination response as well as increased risk of infection. ${ }^{7-9}$ During gestational malaria $(\mathrm{GM})$, the sequestration and cytoadhesion of infected red blood cells within intervillous space of the placenta will lead to local inflammatory responses and alteration of placental functions, and subsequently promote intrauterine growth restriction and prematurity. ${ }^{10}{ }^{11}$ In addition, in utero exposure to Plasmodium falciparum antigens impacts the newborn immune development ${ }^{12} 13$ and is a risk factor predisposing to malaria and other infections during the first year of life. ${ }^{1415}$

Understanding fetal immune maturation and more specifically during the last 3 months of pregnancy is a prerequisite to an accurate interpretation of neonatal response to infection. ${ }^{16} 17$ To respond to pathogens, newborns depend essentially on their innate immune system. The Toll-like receptors (TLRs) represent the first line of defence and play an important role in orchestrating regulatory and inflammatory responses to pathogens and in initiating specific T and B cell responses. ${ }^{17-19}$ Increased risk of early and late neonatal infection and impaired host response seen in premature infants is related to altered innate immune functions. ${ }^{20-22}$ Although the underlying immunological basis are not well studied, small for gestational age (SGA) is also a recognised risk factors of neonatal infections. ${ }^{23}{ }^{24}$ Defective innate immunity seen in premature infants is strongly correlated with the extent of immaturity where extremely low birth weight infants (ie, $<28$ weeks of gestational age) have a merely absent innate immune response. ${ }^{21}$ This state of 'innate' immunodepression, although potentially reversible, shares many similarities to 'acquired' immunodepression seen in critically ill and septic patients. ${ }^{25-27}$ Currently, the most robust and reproducible marker of immunodepression is the measurement of monocytic HLA-DR surface expression (mHLA-DR). Extended literature has correlated its level during the course of disease to 28 days mortality and occurrence of secondary infections. ${ }^{28}$ Recently, two transcriptional biomarkers were shown to identify similarly those conditions. CD74, coding for the $\gamma$ chain (Li chain) of class II CMH and CX3CR1, the fractalkine receptor, were shown to be associated with mortality and/or the occurrence of secondary infections. ${ }^{29}{ }^{30}$ Currently, less data exist on mHLA-DR in neonates. Although not found in all series, mHLA-DR seems to be reduced in premature infants compared with term infants and predicts both early-onset neonatal sepsis (EONS) and late-onset neonatal sepsis (LONS) with an acceptable accuracy (receiver operating characteristic area under the curve 0.78 ; $95 \%$ CI: 0.61 to 0.92$).{ }^{21}{ }^{31}$ No data exist on the value of both CD74 and CX3CR1 transcripts for prognostication of neonatal sepsis. However, current understanding of sepsis pathophysiology and, more specifically, sepsis-induced immunodepression, sustain the exploration of this specific condition, particularly in patients where immunodepressed states are known to be part of the pathophysiology and impact severity. Exploring biomarkers for sepsis diagnosis and prognostication in neonates exposed to GM may prove to be particularly relevant. Both a better characterisation of the immunodepressed state encountered in those patients and optimisation of sepsis diagnosis could be expected by using combined biomarkers, mixing both sepsis diagnosis and immunosuppression identification.

\section{RATIONALE OF THE STUDY}

From a clinical perspective, increased incidence of neonatal sepsis, more specifically to Gram-negative bacteria, has been repetitively shown in sub-Saharan infants. ${ }^{32}$ Effect of GM and prematurity are well known factors associated with bacterial infection susceptibility. ${ }^{133}$ Although several studies have shown that in utero $P$. falciparum exposure impairs the immune development of newborns, ${ }^{34-37}$ making them more likely to develop malaria and other infections in their first months of life, ${ }^{12}$ no study has detailed its immunological impact so far. The increased risk of neonatal sepsis has significant collateral consequences such sepsis overdiagnosis, overuse of broad-spectrum antibiotics and development of antibiotic resistance. In such clinical context, antibiotic stewardship is central but limited by the lack of accurate diagnostic tools. Use of biomarkers of neonatal sepsis in LMIC is limited not only by economic perspectives but also due to the lack of validation in those populations. Only few epidemiological data exploring those conditions exist, as large prospective study requires important clinical and financial support to overcome limited resources in LMIC.

From a biological perspective, both GM and prematurity could have similar impact on the newborn's immune system such as impaired TLRs and T regulatory cells responses explaining this increased susceptibility to infection. ${ }^{34-40}$ Those altered immune responses, similar to a state of immunodepression, may impact the accuracy of biomarkers for EONS. GM-induced and prematurityinduced immunodepression were partially characterised by immune functional assay (cytokines release after ex vivo Pathogens-Associated Molecular Patterns (PAMPs) stimulation) and peripheral blood mononuclear cell (PBMC) phenotyping, but most underlying molecular mechanism still remains to be identified. ${ }^{38}$ Better characterisation of the state of immunosuppression may help in optimising neonatal sepsis diagnosis and management in addition to opening a potential avenue for immune stimulation and restoration of efficient response to pathogens. ${ }^{21}$

\section{Hypothesis}

To overcome the lack of epidemiological data on GM and neonatal sepsis as well as on infant's immune response, the SEPSIS study propose to prospectively follow infants born in an urban and suburban malarial endemic area from the peripartum period to 3 months of age. Biological biomarkers, PBMC phenotyping as well as immune 
functional assays will be performed at delivery and during the follow-up. Reference values will be determined and correlation between the biological measurements and occurrence of clinical neonatal sepsis performed. To explain the increased susceptibility of infants with GM, we hypothesised that in utero exposure to $P$. falciparum antigens modifies the TLRs transcriptional response (specifically of the endotoxins recognition complex myeloid differentiation factor 2: TLR4) and the Treg and Breg function when exposed to Gram-negative bacteria, which would explain the state of immunodepression in their first month of life. Therefore, besides phenotypic and functional characterisation of the immune response, specific analysis of the molecular mechanisms triggering GM-mediated immunodepression will be investigated. The SEPSIS project will provide validated reference values for current and new biomarkers of sepsis and GM-induced immunosuppression in sub-Saharan Africa, and may open new perspective in global understanding of sepsis in immunosuppressed infants.

\section{Aims and objectives}

The primary objective of this study is to evaluate procalcitonin (PCT) for neonatal sepsis diagnosis and evaluate CD74 and CX3CR1 to prognose neonatal sepsis in GM endemic area. The secondary objectives are:

- To evaluate markers association (interleukin (IL)-6, $\mathrm{TNF} \alpha$, IL-10, IP10) to improve diagnosis and prognosis accuracy of PCT, and CD74 and CX3CR1.

- To characterise the epidemiology of neonatal sepsis in this population.

- To characterise the effect of GM on the evolution of the innate and adaptive immune profiles between birth and 3 months of life in infants.
- To evaluate the molecular effect of GM on innate immune response to PAMPs.

The results of this large translational project in subSaharan Africa, where malaria, prematurity/SGA and neonatal infections are major public health issues, will contribute:

- To better understand the consequences of prematurity and GM on the neonatal immune system and the associated immunosuppression.

- To test the use of biomarkers (including immunosuppression biomarkers) to optimise diagnosis and severity assessments of neonatal sepsis in a population with GM-induced immunosuppression and obtain reference values in sub-Saharian Africa.

- To evaluate experimental ex vivo therapy to reverse GM-induced immunosuppression.

\section{METHODS AND ANALYSIS}

SEPSIS is a prospective longitudinal, multicentre observational study conducted in the Abomey-Calavi, Sô-Ava and Cotonou districts in South Benin (figure 1).

\section{Study population}

In this project, patients will be enrolled from (1) three suburban health centres (the suburban arm) in order to follow low-risk delivery and (2) three urban hospitals (the Hospital arm) in order to follow infants born from high-risk delivery. In both arms, only women living in the Abomey-Calavi region will be recruited to facilitate the follow-up and to work on infant from the same geographical area.

- The suburban arm aims to follow low-risk deliveries and includes a part of children born to mothers previously included in the RECIPAL cohort (that aims to

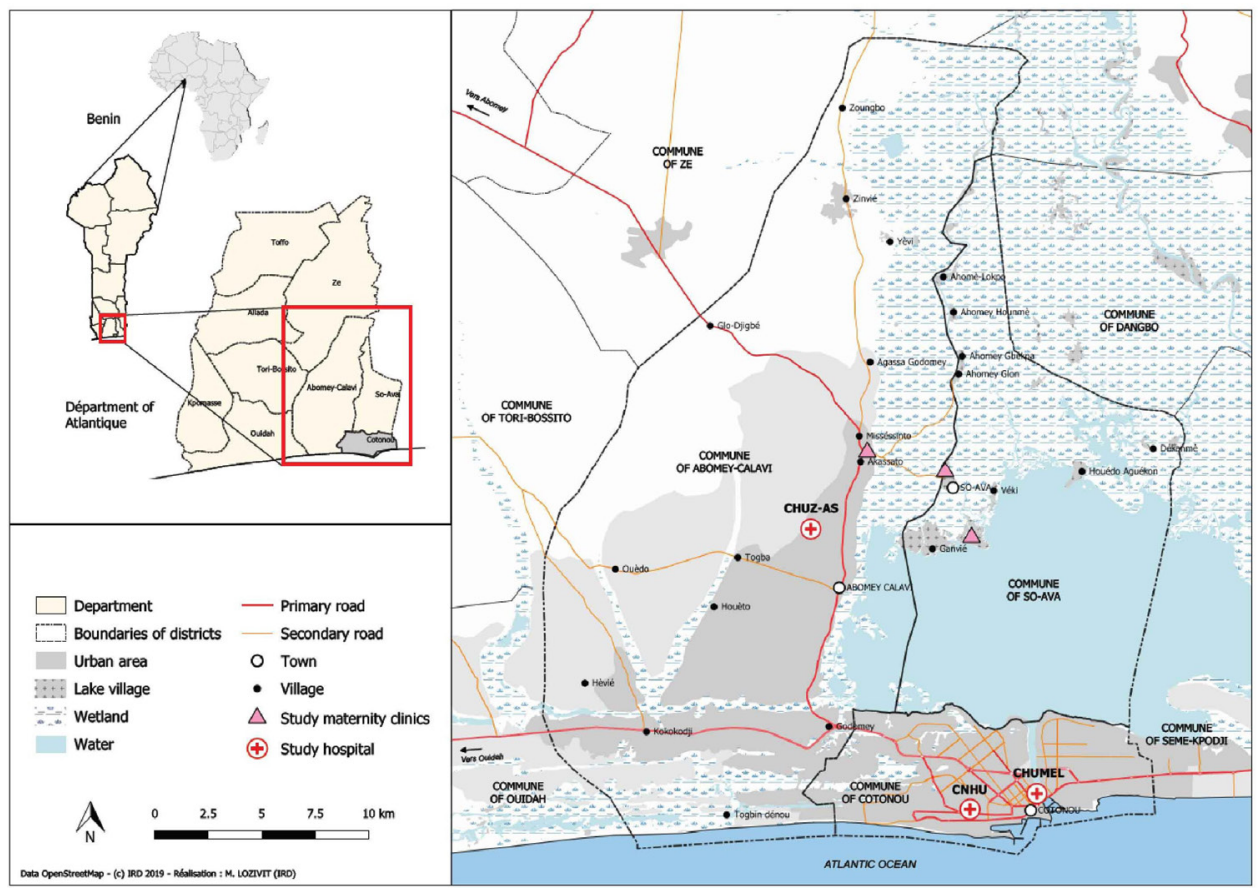

Figure 1 Geographical location of the six study centres in the Sô-Ava, Abomey-Calavi and Cotonou districts in Benin. 
Enrolment

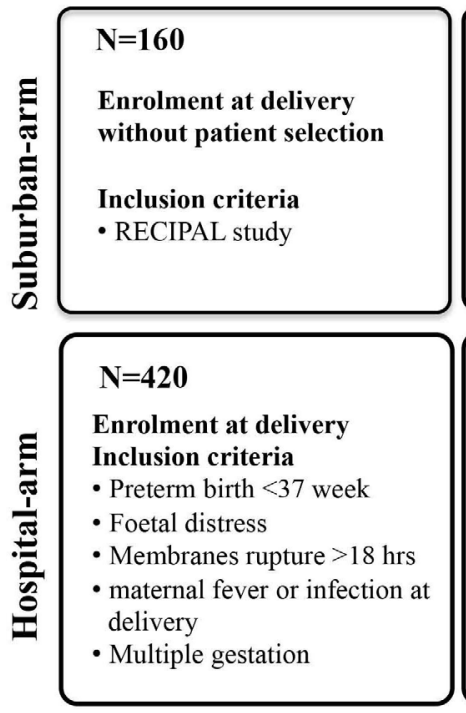

Follow-up and planned analysis

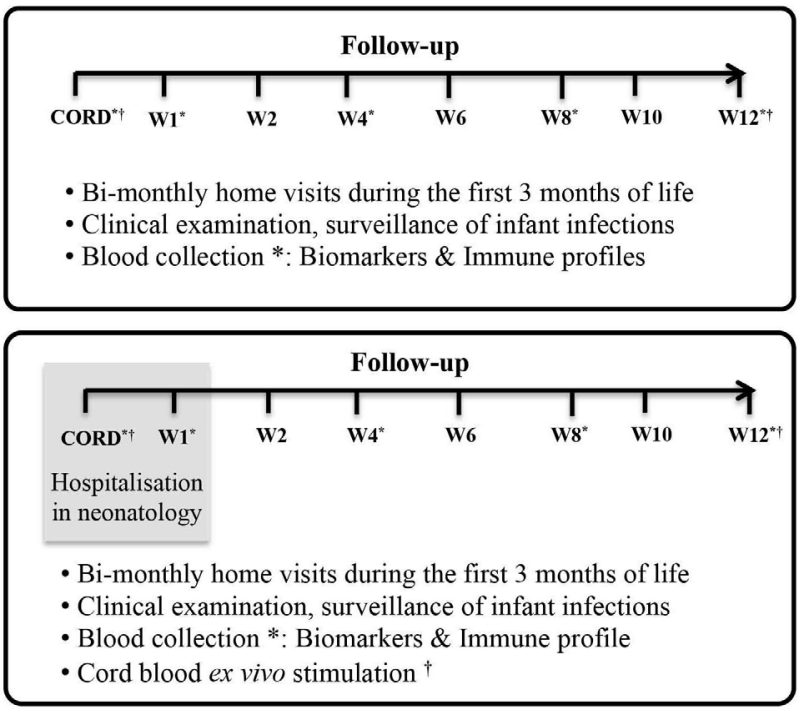

Figure 2 Schematic design of the sepsis study illustrating inclusion, planned biological analysis and follow-up time points.

evaluate the impact of GM on the fetal growth). ${ }^{41}$ In this study arm, out of 411 pregnant women, 273 will be followed until delivery for the study. Because of a delay in the timing between both projects, only the last 160 newborn of the RECIPAL cohort will be enrolled. The expected frequency of GM and prematurity are $40 \%$ and $12 \%$, respectively. This cohort will provide specimen from the child and information on mother's pregnancy and newborn's follow-up.

- The Hospital arm aims to follow children born to mothers with high risk of developing neonatal sepsis. All consecutive deliveries with any perinatal risk of infection were included in the study. Those perinatal risk factors were as follows: prematurity, prolonged ruptures of membranes ( $>18$ hours), maternal fever and/or infection, fetal distress, neonatal resuscitation at delivery and multiple gestation. Children will be recruited at birth in three main university hospitals: Centre Hospitalier Universitaire de la Mère et de l'Enfant Lagune (CHUMEL), Centre National Hospitalier Universitaire (CNHU) in Cotonou and the Centre Hospitalier Universitaire Zone Abomey Calavi / Sô Ava (CHU ZAC) in Calavi. Approximately 420 children will be followed with the inclusion and exclusion criteria presented in figure 2 .

- The study is coordinated by the Institut de Recherche Clinique du Bénin (IRCB).

All children from both arms will be followed clinically on a bimonthly base during the first 3 months of life. The length of the follow-up was decided as being the most risky period in terms of susceptibility to sepsis, neonatal mortality and the development period for the immune responses. $^{17}$

The clinical follow-up of these children will be conducted during the first 12 weeks of life during home visits or in healthcare centres. Newborn from the hospital arm will also be followed in case of hospitalisation for
EONS. We expected that hospitalised newborns will stay from 1 to 10 days at the hospital after birth before going back to their home. The follow-up will consist of scheduled home visits and unscheduled emergency visits if the infant is sick.

1. Newborns will have a full clinical examination by a trained nurse at birth and during home visits (Birth, Week (W)1, W2, W4, W6, W8, W10 and W12).

2. Blood samples will be obtained at birth (cord blood), then at W1, W4, W8 and W12.

3. All sick infants identified during systematic home or unscheduled visits will come for a visit to a healthcare centre, where additional blood sample will be obtained. Unscheduled visits will be called emergency visits in the rest of the document.

4. Healthcare centres are only able to perform haemogram and blood smear. All infants with suspected sepsis needing additional therapeutic and diagnostic workup will be referred to the reference hospitals where sepsis workup can be performed. According to the clinical symptoms, the paediatrician will ask for specific bacteriological analysis to confirm a suspected infection.

\section{Definition and neonatal sepsis diagnosis}

Neonatal sepsis diagnosis will be established by the local paediatrician based on the child clinical examination and initial workup including haemogram, C-reactive protein (CRP) and microbiological cultures (blood, cerebral fluids and urine). In the Hospital arm of the study, neonatal sepsis will be suspected in neonates with perinatal infectious risk factors, as previously described, and more than two of the following criteria being present: neutrophil count $<7500 / \mathrm{mm}^{3}$ or $>14500 / \mathrm{mm}^{3}$, band form $>1500 / \mathrm{mm}^{3}$, immature/total neutrophils ratio $>0.16$, platelets count $<150000 / \mathrm{mm}^{3}$ and CRP $>10 \mathrm{mg} / \mathrm{L}$. Suspected neonatal sepsis will be considered as clinical sepsis when the following clinical signs are associated: 
temperature irregularity; respiratory distress or apnoea; seizures, altered tonus, irritability or lethargy; vomiting, altered feeding pattern, ileus; skin perfusion alteration, haemodynamic signs (tachycardia, hypotension); hypoglycaemic/hyperglycaemic, hyperlactatemia or identification of focal infection such as soft tissue infection or conjunctivitis. In the suburban arm, neonatal sepsis will be suspected if the child demonstrates clinical signs as mentioned. In such case, the child will be transferred to the hospital where further sepsis workup will be performed. Neonatal sepsis will be considered as EONS if occurring within the first 72 hours after delivery, and LONS thereafter. Neonatal sepsis diagnosis and subsequent management will be left at the discretion of the paediatrician in charge of the child. All newborns with a clinical diagnosis of sepsis will be subsequently adjudicated by two independent paediatricians (PT, ULT) and sorted into 'presumed sepsis' and 'definite clinical sepsis'. In addition to the full medical file review, microbiological cultures results will be monitored. Parallel to microbiological culture, we will use the specific BioFire Filmarray panels for all positive blood culture (Blood Culture Identification (BCID) panel) and cerebrospinal fluids (meningitis/encephalitis panel). All studied biomarkers will be kept blinded to the adjudication. In parallel, neonatal risk assessment will be scored using the Neonatal Sepsis Trial Network grid and patients scored from 1 to $3{ }^{42} \mathrm{GM}$ will be defined as a malaria infection during pregnancy or at delivery. For women in the suburban arm, malaria screening will be performed at each scheduled prenatal visit using a thick blood smear. Mothers from the Hospital arm will be screened only at the time of delivery. In this group, antenatal malaria will be established on the basis of mother's anamnesis. For both study arms, placental blood smear and mother's peripheral blood smear will be performed. The Lambaréné technique will be used to quantify parasitaemia with a detection threshold of five parasites per microlitre. $^{43}$

\section{Sample and clinical data collection}

Clinical and biological (biobanking) data dedicated to this project will be gathered according to table 1 .

Table 1 Clinical and biological data collection planning

\begin{tabular}{|c|c|c|c|c|c|c|c|c|c|c|}
\hline & Mother & Birth & W01 & W02 & W04 & W06 & W08 & W10 & W12 & Emergency \\
\hline Inclusion/exclusion criteria & & $x$ & & & & & & & & \\
\hline Demography & $x$ & $x$ & & & & & & & & \\
\hline Reproductive and obstetric & $x$ & & & & & & & & & \\
\hline Malaria in pregnancy & $x$ & & & & & & & & & \\
\hline Clinical examination & $x$ & $x$ & $x$ & $x$ & $x$ & $x$ & $x$ & $\mathrm{x}$ & $x$ & $x$ \\
\hline Temperature & $x$ & $x$ & $x$ & $x$ & $x$ & $x$ & $x$ & $x$ & $x$ & $x$ \\
\hline Pulse rate & & $x$ & $x$ & $x$ & $x$ & $x$ & $x$ & $\mathrm{x}$ & $x$ & $\mathrm{x}$ \\
\hline \multicolumn{11}{|l|}{ Biology } \\
\hline Dry blood spot & $x$ & $x$ & & & $x$ & & $x$ & & $x$ & $x$ \\
\hline Blood smear & $x$ & $x$ & & & $x$ & & $x$ & & $x$ & $x$ \\
\hline Heparin tube sampling & & $x$ & $x$ & & $x$ & & $x$ & & $x$ & $x$ \\
\hline Haemoglobin & & $x$ & $x$ & & $x$ & & $x$ & & $x$ & $x$ \\
\hline TLRs stimulation & & $x$ & & & & & & & $x$ & $x$ \\
\hline Plasma & & $x$ & $x$ & & $x$ & & $x$ & & $x$ & $x$ \\
\hline Procalcitonin & & $x$ & $x$ & & $x$ & & $x$ & & $x$ & $x$ \\
\hline PBMC & & $x$ & & & & & & & $x$ & $x$ \\
\hline
\end{tabular}

PBMC, peripheral blood mononuclear cell; TLR, toll-like receptor; W, weeks. 
For the various biological tests to be performed in this study, the sample collection will allow us to establish several types of biobanking:

1. Heparin plasma for the study of protein biomarkers.

2. Supernatant from unstimulated whole blood and ex vivo stimulated with TLR ligands for the quantitative evaluation of cytokines release.

3. Messenger RNA (mRNA) biobank extracted from cell pellet of unstimulated whole blood and blood stimulated with TLR ligands, preserved in PAXgene (Qiagen, Hilden, Germany) for the study of gene transcripts of TLRs and signalling pathways.

4. mRNA biobank to study transcriptomic host biomarkers (RNA will be extracted from whole blood collected in PAXgene tubes).

5. Biobanking of PBMCs isolated from cord blood and peripheral blood by gradient separation of Ficoll (Sigma, Darmstadt, Germany) density for the exploration of Treg and Breg cells function.

\section{Biomarkers measurements}

This part of the study will consist of testing blood samples of the children from both arms to validate CX3CR1, CD74, IL-10, PCT, IP10, TNF $\alpha$ and IL-6. For transcriptomic biomarkers, after extraction of from whole blood using the QIAsymphony SP/AS automated system (Qiagen, Hilden, Germany) with the QIAsymphony PAXgene blood RNA kit (PreAnalytiX, Hombrechtikon, Switzerland), mRNA from $C D 74$ and $C X 3 C R 1$ will be quantified by quantitative PCR (Q-PCR) using CD74/HPRT1 and CX3CR1/HPRT1 prototype kits (bioMérieux, Marcyl'Etoile, France) on an ABI7500 thermocycler (Thermo Fisher Scientific, Waltham, Massachusetts, USA). Protein biomarkers associated with immunosuppression and inflammation (PCT, IL-6, TNF $\alpha$, IL-10, and IP10) will be measured in heparinised plasma using the multiplex Ella platform (ProteinSimple, San Jose, California, USA).

\section{Dynamic immune profiles}

Here, we plan to study the immune profile of the first 3 months of age. Biological samples will be used to determine and study the transcriptional and functional profiles of the innate and adaptive immunity. We foresee the following analyses:

1. Ex vivo functionality of the innate response via TLRs stimulation. Whole blood stimulation with TLRs agonists (LPS, FSL1, Pam3CSK4, Flagellin, PolyI:C, CPG-ODN, Imiquimod and Resiquimod) (Sigma, Darmstadt, Germany). Proinflammatory and antiinflammatory cytokines/chemokine (IL-6, IL-10, $\mathrm{TNF} \alpha$ ) in supernatants (eBioscience, Thermo Fisher Scientific, Waltham, Massachusetts, USA) and genes transcripts quantification by Q-PCR will be performed using a CFX90 thermocycler (Biorad, Hercules, California, USA). Analysis will be performed in selected patients divided into four groups (GM and sepsis, no GM and sepsis, GM and no sepsis, no GM and no sepsis). Patients will be selected from both study arms.
2. Ex vivo functionality and phenotyping of Treg and Breg lymphocytes. Using flow cytometry (FACS Canto, Becton Dickinson, Franklin Lakes, New Jersey, USA), we will investigate different functional markers like the gut-homing $\alpha 4 \beta 7$ integrins, CD39 and CTLA4 immunosuppressive markers. Second, the frequencies of Treg and Breg producing intracytoplasmic IL-10 and TGF $\beta$ will be assessed in cord blood after mitogen or antigen ex vivo stimulation. ${ }^{44-48}$

\section{Modulation of the host response against $P$. falciparum}

In this part, we aim to design an ex vivo experimental model of co-culture assays to mimic a clinical situation of malaria and Gram-negative infections. The objective is to perform in vitro co-culture functional assays with various stimuli combinations (TLRs agonists and/or $P$. falciparum-schizonte extract) to highlight the mechanisms of endotoxins anergy observed in infants with GM.

\section{Sample size and data analysis plan}

To assess the accuracy of new biomarkers of sepsis (PCT) and immunosuppression (CD74 and CX3CR1): we estimated that of the 400 children, 40 would get an infection (fever) within 7 days after birth and 189 would get an infection within 3 months of follow-up (W2 to W12) after birth. With an expected proportion of $75 \%$ infection in PCT + and 25\% in PCT- (knowing that the difference in proportions is probably even greater between the two groups), t68 subjects (34 in each group) will be required to reach a $99 \%$ power and risk of first kind of $5 \%$, which shows that the size of this study (higher in both groups) will answer this question with a power reaching almost $100 \%$. Statistical analyses were performed using R software V.3.6.1. Numbers and frequency were used for categorical data and means (SD) or medians (IQR) for continuous data. Categorical variables were compared using the $\chi^{2}$ test (or Fisher's exact test for small expected numbers). The distribution of continuous data was compared using Student's t-test (or the Mann-Whitney test when distribution was not normal) if the outcome was two groups, and analysis of variance (or the Kruskal-Wallis test when distribution was not normal) if not. OR and $95 \%$ CI were assessed using logistic regression models. An adjustment to the $p$ values ( $q$ value) was performed using False Discovery Rate (FDR) correction (Benjamini-Hochberg). The independent variables initially included in the model were those associated with the infection of the newborn or infant in univariate analysis with $\mathrm{p}<20 \%$ and a stepwise or backward procedure was applied to assess variables that were significantly and independently associated with the infection of the newborn or infant at $5 \%$ level. When the linearity hypothesis was not respected, the continuous variables were transformed into ordered data (clinically meaningful classes or median separation). To analyse the infection of the newborn or infant, we considered variables associated with the patient (history of maternal infections, gestational age, child's age, birth weight, prematurity, GM, SGA and other risk factors) and/or 
with the different biomarkers (PCT, CD74 and CX3CR1). All reported $\mathrm{p}$ values are two-sided and the significance threshold was set at $<0.05$. Other machine models (Random Forest, Decision Tree, Support Vector Machine and k-Nearest Neighbour) will be used to increase performance. For this purpose, the data will be divided into two parts: a training part (algorithm training) and a test part (to obtain performance).

\section{ETHICS AND DISSEMINATION \\ Ethics approval}

The protocol, information documents and consent forms for legal representatives received approval from the ethical committee (CER-ISBA) in Benin (Approval code: CER-ISBA 85 - 5 April 2016). An amendment has been filed to extend the study to a second hospital cohort. This amendment was approved on 3 February 2017). This study complies with the Declaration of Helsinki principles of Good Practice and the Nagoya protocol.

\section{Informed consent}

- The study investigator who identified the child and the parents of the child will read the information document aloud and give them full details in the event of any questions.

- If the child's parents are literate, the information will be administered in French, and consent will be given to one of the parents (father or mother) of the child for proofreading. After reading and answering any questions from parents, the consent form will be signed. One of the parents and the interviewer will complete and sign the form in duplicate. One copy will be given to the parents and the second copy will be placed in the master file of the study.

- If the child's parents are illiterate, the information will be administered in the local language (Mainly, Fon, Toffin, Aizo or other). In addition, the information document will be given to an impartial witness, independent of the investigation and previously identified by the parents themselves, who will act as a signatory witness. This person may be a parent or guardian. After the parents' questions have been answered, consent will be signed: one of the parents will put the fingerprint of the left index finger in place of the signature and the witness present throughout the parent information process will sign the consent to his turn. Finally, the investigator will complete and sign the consent. The form will be completed in two copies. The parents will keep a copy of the consent and the second copy will be kept in the master file of the study.

- The consents will be kept at the IRCB premises for 5 years.

\section{Safety of participants}

This study includes no serious foreseeable risk to the health of the children involved. The only potential risk is related to blood sample collection (maximum total blood per sampling $1 \mathrm{~mL} / \mathrm{kg}$, collected over all time points). In cases where children develop sepsis requiring hospitalisation, all related costs will be supported by the investigators.

\section{Study management}

The study is coordinated by bioMérieux and a dedicated team composed from members of the entire consortium. The clinical trial was coordinated by the IRCB.

\section{Data management and handling}

For each patient, a paper Case Report Form (CRF) including sociodemographic, anthropometric, clinical and paraclinical information will be completed by clinical assistants and healthcare providers. MS Access was used to gather all the data from this clinical study. RStudio V.1.1.383 (RStudio, Boston, Massachusetts, USA) will be used to perform the statistical analysis of data. All samples and initial processing of the biobanking will be performed at the IRCB. Subsequently, biobanking will either stay at the IRCB or be transferred to the Christophe Mérieux research centre in Grenoble, France, for further processing (CD74 and CX3CR1 Q-PCR; biomarkers dosing). Whole blood ex vivo stimulation will be transferred to the Institute of Integrative Biology of the Cell, Gif-sur-Yvette, France. Data set will be available from the IRCB.

\section{Medical confidentiality}

All the data collected will be pseudonymised and covered by medical confidentiality. To protect the participant's confidentiality, samples will be de-identified. The investigator will ensure that confidentiality is maintained. Patients will only be identified by their initials and study number (ID code) on the observation books or on all other documents. These ID codes will be kept separately. Patient data, identified by the ID code will be stored separately. The investigator will ensure the retention of the identification list of patients included in the study. This includes at least the first page of the CRF and the Access databases (Microsoft, Redmond, Washington, USA). All the CRFs will be kept in the IRCB premises for at least 5 years in an air-conditioned container. As part of data processing, data consultation and data analysis performed in France, all patients at each site will be informed of the data processing procedure as well as their rights including the right of access to and the right of opposition to the transmission of the data.

Professional secrecy will be respected by all the persons participating in the conduct of this study. The manager, the investigator and all their collaborators undertake the responsibility to not transmit to a third party any of the confidential information obtained through this research, including information on the health status of the patients or about the current study.

\section{Author affiliations}

${ }^{1}$ Institut de Recherche pour le Développement (IRD), Mère et enfant face aux infections tropicales (UMR216), Paris, France 
${ }^{2}$ COMUE Sorbonne Paris Cité, Universite Paris Descartes, Paris, Île-de-France, France

${ }^{3}$ Department of Microbiology, Institut de Biologie Integrative de la Cellule, Gif-surYvette, France

${ }^{4}$ Medical Diagnostic Discovery Department (MD3), bioMerieux SA, Marcy l'Etoile, Rhône-Alpes, France

${ }^{5}$ UMR216-MERIT, French National Research Institute for Sustainable Development (IRD), Université Paris Descartes, Paris, France

${ }^{6}$ Institut de Recherche Clinique du Bénin, Calavi, Benin

${ }^{7}$ Institut de Recherche Clinique du Benin, Cotonou, Benin

${ }^{8}$ UMR216, Institut de Recherche pour le Développement, Cotonou, Benin

${ }^{9}$ Faculté de Pharmacie, Université Paris Descartes, Paris, France

${ }^{10}$ EA 7426 Pathophysiology of Injury-Induced Immunosuppression, bioMerieux, LYON cedex 03, France

${ }^{11}$ Département d'Anesthésie et de Réanimation, Hospices Civils de Lyon, LYON

Cedex 03, France

${ }^{12}$ Pediatric Intensive Care, Hopitaux Universitaires Paris-Sud, Le Kremlin-Bicetre, France

${ }^{13}$ Faculté des Sciences de la Santé (FSS), Cotonou, Benin

${ }^{14}$ Department of Paediatric, National University Hospital Center (CNHU), Cotonou,

Benin

${ }^{15}$ Department of Paediatric, Centre Hospitalier Universitaire de la Mère et de l'Enfant Lagune (CHUMEL), Cotonou, Benin

${ }^{16}$ Institut de Recherche Clinique du Benin, Calavi, Île-de-France, Benin

${ }^{17}$ CHU-MEL Hospital, Cotonou, Benin

${ }^{18}$ Calavi Hospital, Calavi, Benin

\section{Twitter Pierre Tissieres @PierreTissieres}

Acknowledgements We thank the entire RECIPAL and SEPSIS team, including research scientists, engineers, technicians and managers.

Collaborators SEPSIS study group collaborators: Aurax Fernando, Urbain Ahouayito, Basile Agossou, Caleb Ezinmegnon, Anauel Fortunato, Josué Fiagbenou, Djamirou Dossa, Dramane Abdou, Canisius Fantodji, Nawal Sare, Wilisto Bara, Razack Monde, Erasme Gbagidi, Larissa Allokpe, Armand Housemenou, Landry Assongba, Manfred Accrombessi, Florent Kouhouenou, Armand Hounsemenou, Amour Ridagba, Christiane Aguemon, Dissou Affolabi, Réné Perrin, Benjamin Hounkpatin, Dossou Dagba, Luis Augusto, Sophie Thibault, François Bartolo, Marine Mommert and Karen Brengel-Pesce

Contributors NF, JYM, AP, VB and PT conceived and designed the study. NF, GA, SE, RL, KG, DS, AM, LB, NT, Md, JA and IDD managed infants follow-up in the field hospital and in the lab. SE, NF, SB, GC, UL-T and PT analysed the data. NF, GA, SE, $\mathrm{RL}, \mathrm{KG}$ and DS contributed reagents/materials/analysis tools. NF, SE and PT drafted and finalised the manuscript. LV, AP, JT, GA, VB and PT revised the manuscript. The final manuscript was read and approved by all authors.

Funding The project is funded by a consortium: bioMérieux, IRD and the CNRS. We are extremely grateful to all families who took part in this study, and to midwifes, nurses and community health workers for recruiting and following families up. SE was co-funded by CIFRE PhD grant (CIFRE number 2016/1550) and bioMérieux. GA was funded by the Fondation pour la Recherche Médicale (FRM grant number EC020160736054 to GA) for a PhD scholarship.

Map disclaimer The depiction of boundaries on this map does not imply the expression of any opinion whatsoever on the part of BMJ (or any member of its group) concerning the legal status of any country, territory, jurisdiction or area or of its authorities. This map is provided without any warranty of any kind, either express or implied.

Competing interests JYM, LV, JT, AP and SB are employed by an in vitro diagnostic company, bioMérieux SA. The remaining authors declare that this research was conducted in the absence of any commercial or financial relationship that could cause potential conflict of interest.

Patient and public involvement Patients and/or the public were involved in the design, or conduct, or reporting, or dissemination plans of this research. Refer to the Methods section for further details.

Patient consent for publication Not required.

Provenance and peer review Not commissioned; externally peer reviewed.

Open access This is an open access article distributed in accordance with the Creative Commons Attribution Non Commercial (CC BY-NC 4.0) license, which permits others to distribute, remix, adapt, build upon this work non-commercially, and license their derivative works on different terms, provided the original work is properly cited, appropriate credit is given, any changes made indicated, and the use is non-commercial. See: http://creativecommons.org/licenses/by-nc/4.0/.

\section{ORCID iDs}

Julien Textoris http://orcid.org/0000-0002-3821-9337

Pierre Tissieres http://orcid.org/0000-0001-5423-5532

\section{REFERENCES}

1 Seale AC, Blencowe $\mathrm{H}$, Manu AA, et al. Estimates of possible severe bacterial infection in neonates in sub-Saharan Africa, South Asia and Latin America for 2012: a systematic review and meta-analysis. Lancet Infect Dis 2014:14:731-41.

2 Blencowe $\mathrm{H}$, Cousens S. Addressing the challenge of neonatal mortality. Trop Med Int Health 2013;18:303-12.

3 Blencowe $\mathrm{H}$, Cousens S, Oestergaard MZ, et al. National, regional, and worldwide estimates of preterm birth rates in the year 2010 with time trends since 1990 for selected countries: a systematic analysis and implications. Lancet 2012;379:2162-72.

4 Katz J, Wu LA, Mullany LC, et al. Prevalence of small-for-gestationalage and its mortality risk varies by choice of birth-weight-forgestation reference population. PLoS One 2014;9:e92074.

5 Bailey PE, Andualem W, Brun M, et al. Institutional maternal and perinatal deaths: a review of 40 low and middle income countries. BMC Pregnancy Childbirth 2017;17:295.

6 Ranjeva SL, Warf BC, Schiff SJ. Economic burden of neonatal sepsis in sub-Saharan Africa. BMJ Glob Health 2018;3:e000347.

7 Labeaud AD, Malhotra I, King MJ, et al. Do antenatal parasite infections devalue childhood vaccination? PLoS Negl Trop Dis 2009;3:e442.

8 Dauby N, Goetghebuer T, Kollmann TR, et al. Uninfected but not unaffected: chronic maternal infections during pregnancy, fetal immunity, and susceptibility to postnatal infections. Lancet Infect Dis 2012;12:330-40.

9 Sylvester B, Gasarasi DB, Aboud S, et al. Prenatal exposure to Plasmodium falciparum increases frequency and shortens time from birth to first clinical malaria episodes during the first two years of life: prospective birth cohort study. Malar J 2016;15:379.

10 Briand V, Le Hesran J-Y, Mayxay M, et al. Prevalence of malaria in pregnancy in southern Laos: a cross-sectional survey. Malar $J$ 2016;15:436.

11 Accrombessi M, Fievet N, Yovo E, et al. Prevalence and associated risk factors of malaria in the first trimester of pregnancy: a preconceptional cohort study in Benin. $J$ Infect Dis 2018;217:1309-17.

12 Odorizzi PM, Feeney ME. Impact of in utero exposure to malaria on fetal T cell immunity. Trends Mol Med 2016;22:877-88.

13 Barboza R, Hasenkamp L, Barateiro A, et al. Fetal-derived MyD88 signaling contributes to poor pregnancy outcomes during gestational malaria. Front Microbiol 2019;10:68.

14 Le Hesran JY, Cot M, Personne P, et al. Maternal placental infection with plasmodium falciparum and malaria morbidity during the first 2 years of life. Am J Epidemiol 1997;146:826-31.

15 Rachas A, Le Port A, Cottrell G, et al. Placental malaria is associated with increased risk of nonmalaria infection during the first 18 months of life in a beninese population. Clin Infect Dis 2012;55:672-8.

16 Veru F, Laplante DP, Luheshi G, et al. Prenatal maternal stress exposure and immune function in the offspring. Stress 2014;17:133-48

17 Yu JC, Khodadadi H, Malik A, et al. Innate immunity of neonates and infants. Front Immunol 2018;9:9.

18 Luciano AA, Arbona-Ramirez IM, Ruiz R, et al. Alterations in regulatory $\mathrm{T}$ cell subpopulations seen in preterm infants. PLoS One 2014;9:e95867.

19 Sharma AA, Jen R, Brant R, et al. Hierarchical maturation of innate immune defences in very preterm neonates. Neonatology 2014:106:1-9.

20 Lavoie PM, Huang Q, Jolette E, et al. Profound lack of interleukin (IL)$12 / \mathrm{IL}-23 \mathrm{p} 40$ in neonates born early in gestation is associated with an increased risk of sepsis. J Infect Dis 2010;202:1754-63.

21 Tissières $P$, Ochoda A, Dunn-Siegrist I, et al. Innate immune deficiency of extremely premature neonates can be reversed by interferon- $\gamma$. PLoS One 2012;7:e32863.

22 Glaser K, Speer CP. Toll-Like receptor signaling in neonatal sepsis and inflammation: a matter of orchestration and conditioning. Expert Rev Clin Immunol 2013;9:1239-52. 
23 Stoll BJ, Hansen NI, Bell EF, et al. Neonatal outcomes of extremely preterm infants from the NICHD neonatal research network. Pediatrics 2010;126:443-56.

24 Tröger B, Müller T, Faust K, et al. Intrauterine growth restriction and the innate immune system in preterm infants of $\leq 32$ weeks gestation. Neonatology 2013;103:199-204.

25 Boomer JS, To K, Chang KC, et al. Immunosuppression in patients who die of sepsis and multiple organ failure. JAMA 2011;306:2594-605.

26 Winkler MS, Rissiek A, Priefler M, et al. Human leucocyte antigen (HLA-DR) gene expression is reduced in sepsis and correlates with impaired TNF $\alpha$ response: a diagnostic tool for immunosuppression? PLoS One 2017;12:e0182427.

27 Payen D, Faivre V, Miatello J, et al. Multicentric experience with interferon gamma therapy in sepsis induced immunosuppression. A case series. BMC Infect Dis 2019;19:931.

28 Venet F, Lukaszewicz A-C, Payen D, et al. Monitoring the immune response in sepsis: a rational approach to administration of immunoadjuvant therapies. Curr Opin Immunol 2013;25:477-83.

29 Pachot A, Cazalis M-A, Venet F, et al. Decreased expression of the fractalkine receptor CX3CR1 on circulating monocytes as new feature of sepsis-induced immunosuppression. J Immunol 2008;180:6421-9.

30 Peronnet E, Venet F, Maucort-Boulch D, et al. Association between mRNA expression of CD74 and IL10 and risk of ICU-acquired infections: a multicenter cohort study. Intensive Care Med 2017;43:1013-20.

31 Palojärvi A, Petäjä J, Siitonen S, et al. Low monocyte HLA-DR expression as an indicator of immunodepression in very low birth weight infants. Pediatr Res 2013;73:469-75.

32 Medugu N, Iregbu K, Iroh Tam P-Y, et al. Aetiology of neonatal sepsis in Nigeria, and relevance of group B Streptococcus: a systematic review. PLoS One 2018;13:e0200350.

33 Agbota G, Polman K, Wieringa FT, et al. Maternal malaria but not schistosomiasis is associated with a higher risk of febrile infection in infant during the first 3 months of life: a mother-child cohort in Benin PLoS One 2019;14:e0222864

34 Gbédandé K, Varani S, Ibitokou S, et al. Malaria modifies neonatal and early-life Toll-like receptor cytokine responses. Infect Immun 2013;81:2686-96.

35 Natama HM, Rovira-Vallbona E, Somé MA, et al. Malaria incidence and prevalence during the first year of life in Nanoro, Burkina Faso: a birth-cohort study. Malar J 2018;17:163.

36 Adegnika AA, Köhler C, Agnandji ST, et al. Pregnancy-Associated malaria affects Toll-like receptor ligand-induced cytokine responses in cord blood. J Infect Dis 2008;198:928-36.
37 Nouatin O, Gbédandé K, Ibitokou S, et al. Infants' peripheral blood lymphocyte composition reflects both maternal and post-natal infection with Plasmodium falciparum. PLoS One 2015;10:e0139606.

38 Brustoski K, Kramer M, Möller U, et al. Neonatal and maternal immunological responses to conserved epitopes within the DBLgamma3 chondroitin sulfate A-binding domain of Plasmodium falciparum erythrocyte membrane protein 1. Infect Immun 2005;73:7988-95.

39 Franklin BS, Parroche P, Ataíde MA, et al. Malaria primes the innate immune response due to interferon-gamma induced enhancement of toll-like receptor expression and function. Proc Natl Acad Sci USA 2009;106:5789-94.

40 Mackroth MS, Malhotra I, Mungai P, et al. Human cord blood CD4+CD25hi regulatory $T$ cells suppress prenatally acquired $T$ cell responses to plasmodium falciparum antigens. J Immunol 2011;186:2780-91.

41 Accrombessi M, Yovo E, Cottrell G, et al. Cohort profile: effect of malaria in early pregnancy on fetal growth in Benin (RECIPAL preconceptional cohort). BMJ Open 2018;8:e019014.

42 Stocker M, van Herk W, El Helou S, et al. Procalcitonin-guided decision making for duration of antibiotic therapy in neonates with suspected early-onset sepsis: a multicentre, randomised controlled trial (NeoPIns). Lancet 2017;390:871-81.

43 Swysen C, Vekemans J, Bruls M, et al. Development of standardized laboratory methods and quality processes for a phase III study of the RTS, S/AS01 candidate malaria vaccine. Malar J 2011;10:223

44 Lundell A-C, Johansen S, Adlerberth I, et al. High proportion of CD5+ $B$ cells in infants predicts development of allergic disease. $J$ Immunol 2014;193:510-8.

45 Lundell A-C, Hesselmar B, Nordström I, et al. Higher B-cell activating factor levels at birth are positively associated with maternal dairy farm exposure and negatively related to allergy development. $J$ Allergy Clin Immunol 2015;136:e1073:1074-82.

46 Rueda CM, Moreno-Fernandez ME, Jackson CM, et al. Neonatal regulatory $T$ cells have reduced capacity to suppress dendritic cell function. Eur J Immunol 2015;45:2582-92.

47 Rueda CM, Wells CB, Gisslen T, et al. Effect of chorioamnionitis on regulatory $\mathrm{T}$ cells in moderate/late preterm neonates. Hum Immunol 2015;76:65-73

48 Rennó C, Nadaf MIV, Zago CA, et al. Healthy preterm newborns show an increased frequency of CD4(+) CD25(high) CD127(low) FOXP3(+) regulatory $T$ cells with a naive phenotype and high expression of gut-homing receptors. Scand J Immunol 2016;83:445-55. 